\title{
Raman-scattering criteria for characterization of anneal-restored zinc blende single crystals: Application to $\mathrm{Si}^{+}$-implanted $\mathrm{InP}$
}

\author{
L. Artús, ${ }^{a}$ R. Cuscó, and J. Ibáñez \\ Insitut Jaume Almera, Consell Superior d'Investigacions Científiques (CSIC), Lluís Solé i Sabarís s.n., \\ 08028 Barcelona, Spain
}

\author{
J. M. Martin and G. González-Díaz \\ Departamento de Electricidad y Electrónica, Facultad de Física, Universidad Complutense, \\ 28040 Madrid, Spain
}

(Received 17 April 1997; accepted for publication 11 July 1997)

\begin{abstract}
We have studied the lattice recovery by rapid thermal annealing of $\mathrm{Si}^{+}$-implanted $\mathrm{InP}$ using Raman spectroscopy. The crystallinity recovery for different annealing temperatures of samples totally amorphized by the implantation can be monitored by means of their Raman spectra. However, free-charge coupling with the LO mode and possible misorientation of the recrystallized material may alter substantially the first-order Raman spectrum, making it unreliable for a good characterization of the lattice recovery. The study of second-order Raman spectrum overcomes the problems present in the analysis of first-order Raman spectrum and provides suitable criteria to assess the recrystallization of the implanted and annealed samples. After rapid thermal annealing at $875^{\circ} \mathrm{C}$ for $10 \mathrm{~s}$, the intensity of the second-order peaks approaches $70 \%$ of its value in virgin InP, and third-order Raman peaks are also clearly detected, evidencing the good lattice recovery achieved. (C) 1997 American Institute of Physics. [S0021-8979(97)03820-6]
\end{abstract}

\section{INTRODUCTION}

Ion implantation is one of the main techniques for selective doping of semiconductors, as it provides good control on dopant concentration and profile. However, the implantation process damages the crystal structure, and may even lead to total amorphization depending on the type of ion and implantation conditions. Postimplantation annealing treatments are necessary to achieve lattice recovery and electric activation of the dopants. The determination of the optimal annealing conditions for which the highest values of activation and carrier mobility are obtained is a common topic in the studies of implanted semiconductors. These studies show that high values of electrical activation and carrier mobility require the incorporation of the dopants into the substitutional lattice sites as well as the removal of traps and defects of the lattice, ${ }^{1}$ and, therefore, a high degree of lattice recovery. Although other techniques such as Rutherford backscattering or transmission electron microscopy can be used to probe lattice disorder, they are destructive in nature. Therefore, Raman scattering emerges as the most useful nondestructive technique to assess lattice recovery.

A large number of articles can be found on Raman scattering of implanted GaAs dealing with lattice damage after implantation $^{2-9}$ as well as lattice recovery by subsequent annealing. ${ }^{10-15}$ By contrast, only a few studies on Raman scattering of ion-implanted $\mathrm{InP}^{3,15-17}$ can be found, in which $\mathrm{Si},{ }^{15} \mathrm{Ga},{ }^{16} \mathrm{Zn},{ }^{17}$ and $\mathrm{Be}^{3,15}$ ions were implanted. Leaving aside a few studies on $\mathrm{GaAs}^{11-13}$ in which rapid thermal annealings (RTA) were performed, only conventional furnace annealings were carried out, and the activation rates obtained were lower compared to RTA, particularly for high implantation doses. ${ }^{18}$ Apart from Refs. 12 and 17, lattice

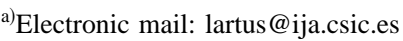

recovery after annealing was assessed in these studies by means of first-order Raman scattering, associating the presence of first-order Raman peaks in the spectra with crystalline recovery. As we shall discuss below, first-order Raman peaks are not well suited to characterize the degree of recrystallization of implanted, (100)-oriented zinc blende semiconductors. Regarding Refs. 12 and 17, in Ref. 12 the secondorder Raman spectrum of an annealed sample was measured, but its shape was substantially different from that of unimplanted InP, whereas in Ref. 17 the intensity of the $2 \mathrm{LO}$ peak was normalized to that of the first-order TO peak, thus introducing into the analysis of the second-order peaks the same problems present in the first-order spectra. Leaving aside the criteria used in these studies, the spectra reported in the articles mentioned above $\mathrm{e}^{3,10-17}$ indicate a poor lattice recovery of the samples implanted at high doses.

The aim of the present article is to show that first-order Raman scattering is not enough to ascertain the quality of the lattice recovery, as well as to discuss the criteria which can provide a more accurate information about the degree of lattice recovery. Using these criteria, we show that for $\mathrm{Si}^{+}$-implanted $\mathrm{InP}$ a good lattice recovery is achieved by RTA, even in samples which were fully amorphized by the implantation process.

\section{EXPERIMENT}

Sample (100) wafers of semi-insulating, Fe-doped InP supplied by Sumitomo were used in this work. The samples were implanted with ${ }^{28} \mathrm{Si}^{+}$at $150 \mathrm{keV}$ at a dose of $5 \times 10^{14}$ $\mathrm{cm}^{-2}$. For these implantation conditions we have previously reported the full amorphization of the InP crystal. ${ }^{19}$ In InP, higher $\mathrm{Si}^{+}$implantation doses give rise to a dramatic decrease of the activation rate, ${ }^{18}$ which is attributed to the amphoteric behavior of $\mathrm{Si}$ in the InP lattice. ${ }^{18,20}$ Subsequent 
RTA annealings for $10 \mathrm{~s}$ at temperatures ranging from 300 to $875^{\circ} \mathrm{C}$ were performed using a RTP-600 system from MPT Corp. in a graphite susceptor, face down on a $\mathrm{Si}$ wafer. We have found that annealings at temperatures higher than $875^{\circ} \mathrm{C}$ produce a deterioration of the surface morphology, in agreement with previous reports. ${ }^{18}$ Electrical measurements performed on samples annealed at $875^{\circ} \mathrm{C}$ for $10 \mathrm{~s}$ showed activation of about $60 \%$ and electronic mobility of about $1000 \mathrm{~cm}^{2} \mathrm{~V}^{-1} \mathrm{~s}^{-1},{ }^{21}$ in good agreement with previously reported data on similar implantation and annealing conditions. ${ }^{18}$

The Raman measurements were performed using the $514.5 \mathrm{~nm}$ line of an $\mathrm{Ar}^{+}$laser, with a power on the sample of about $150 \mathrm{~mW}$. For this wavelength, taking into account the corresponding value of the absorption coefficient of $\mathrm{InP},{ }^{22}$ we estimate that about $10 \%$ of the initial intensity is still acting as probing light at a depth of $100 \mathrm{~nm}$. The Raman spectra were recorded at room temperature using a JobinYvon spectrometer equipped with a charge-coupled device detector cooled with liquid nitrogen. The experiments were performed in backscattering configuration on a (100) face. First-order Raman spectra were recorded using the tripleadditive configuration of the spectrometer, with an entrance slit of $100 \mu \mathrm{m}$, whereas second- and third-order Raman spectra were recorded using the double-subtractive configuration of the spectrometer, with $100 \mu \mathrm{m}$ slits. Prior to the Raman scattering measurements, the surface of the samples was etched in a $\mathrm{H}_{2} \mathrm{O}: \mathrm{H}_{3} \mathrm{PO}_{4}$ solution for 1 min, rinsed with de-ionized water and methanol and blown dry with argon.

\section{RESULTS AND DISCUSSION}

Previous studies of lattice recovery of implanted $\mathrm{GaAs}^{10-15}$ and $\mathrm{InP}^{15,17}$ used the presence of first-order peaks to check the sample crystallinity. In the present article we show that this procedure does not suffice to establish the optimal recrystallization. Firstly, in $n$-type samples implanted at high doses a high concentration, high-mobility free-electron gas is formed after annealing. Then, the presence of an intense, well-defined $L^{-}$coupled-mode peak at a frequency asymptotically close to the TO frequency may induce to confusion in the analysis of the intensity of the TO peak. Even in the cases where the long-wavelength $L^{-}$ modes are not detected, as in $p$-type implanted samples, ${ }^{17,23}$ the intensity of the first-order TO mode is not a suitable reference because this mode is forbidden in backscattering on a (100) face. Secondly, the LO peak observed in these samples arises from Raman scattering in the surface depletion zone of the sample. The associated scattering volume is much smaller than in unimplanted samples, and therefore comparisons of the intensity of the first-order LO peak between implanted and unimplanted samples cannot be used to assess the degree of lattice recovery. Thirdly, a poor annealing may result in samples with a significant proportion of polycrystalline and/or misoriented crystalline regions. In this case, the TO intensity would be much higher compared to the forbidden TO mode of the unimplanted (100)-oriented crystal, and therefore an increase of the TO intensity cannot be considered as indicative of improvement of crystallinity.

Figure 1 displays the first-order Raman spectra of

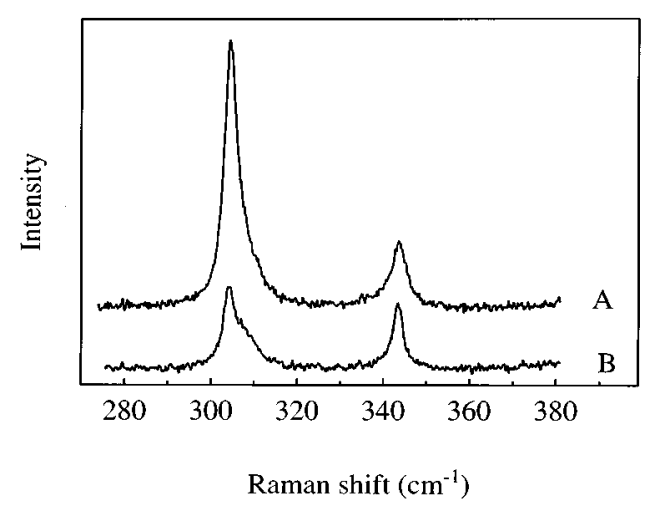

FIG. 1. Room temperature first-order Raman spectra of a $\mathrm{Si}^{+}$-implanted InP sample with a dose of $5 \times 10^{14} \mathrm{~cm}^{-2}$ and annealed at 700 (curve A) and 875 ${ }^{\circ} \mathrm{C}$ (curve B) using RTA. The spectra were recorded in the $X\left(Y^{\prime} Z^{\prime}\right) \bar{X}$ configuration.

samples annealed at 700 and $875^{\circ} \mathrm{C}$ measured in $X\left(Y^{\prime} Z^{\prime}\right) \bar{X}$ configuration, where $X, Y^{\prime}$, and $Z^{\prime}$ are defined along the [100], [011], and [011] directions, respectively. According to selection rules, in this polarization configuration firstorder TO and LO phonons as well as LO-plasmon coupled modes are forbidden ${ }^{23}$ and thus a possible contribution of the $L^{-}$mode is avoided. As can be seen in Fig. 1, the intensity of the TO mode for the sample annealed at $700{ }^{\circ} \mathrm{C}$ is much higher than for the sample annealed at $875^{\circ} \mathrm{C}$. Taking into account that the TO is forbidden in this configuration, the spectra of Fig. 1 suggest that a much better recovery of the (100)-oriented single crystal is achieved in the sample annealed at $875^{\circ} \mathrm{C}$ than in the sample annealed at $700{ }^{\circ} \mathrm{C}$, in which a sizable degree of misorientation and/or polycrystalline regions are still present.

Since other factors apart from the lattice recovery contribute to the changes in the first-order Raman spectra, higher order Raman spectroscopy becomes necessary to assess the quality of the lattice recovery. Figure 2 shows unpolarized second- and third-order Raman spectra of a sample annealed at $875^{\circ} \mathrm{C}$, where the intensity of the second-order peaks is recovered to about $70 \%$ of its value in unimplanted samples. To our knowledge, no third-order Raman scattering had been previously reported on an implanted and subsequently an-

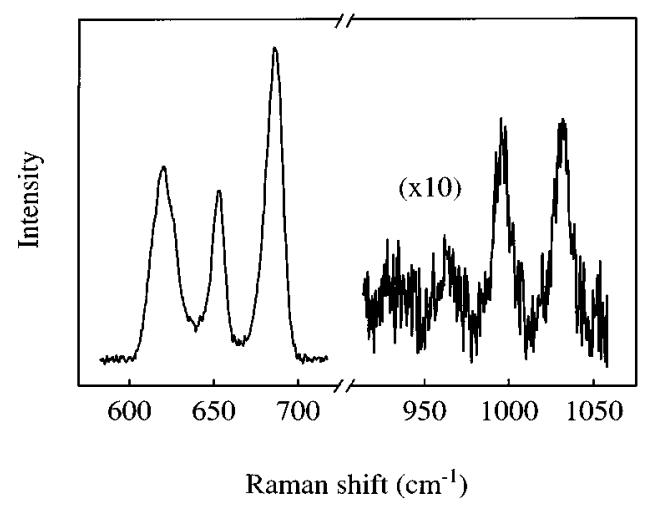

FIG. 2. Second- and third-order Raman spectra of $\mathrm{Si}^{+}$-implanted $\mathrm{InP}$ samples annealed by RTA at $875^{\circ} \mathrm{C}$. 


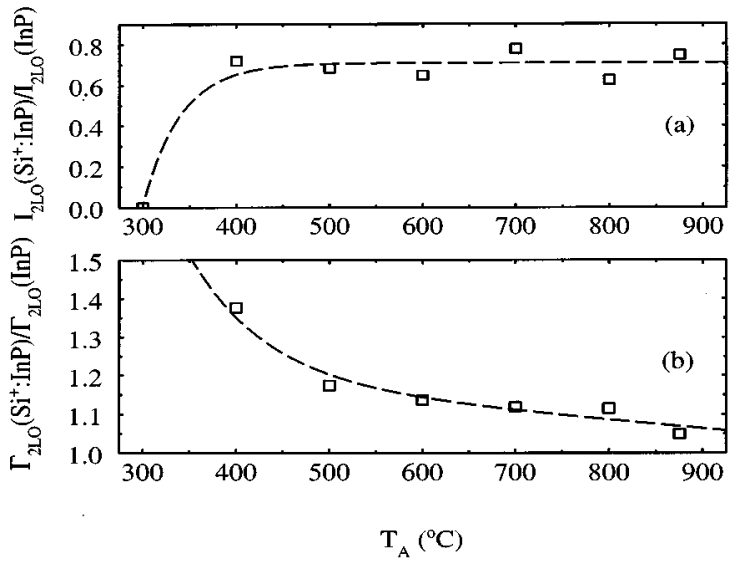

FIG. 3. Plot of $2 \mathrm{LO}$ (a) intensity and (b) width vs RTA annealing temperature. The dashed line is a guide to the eye.

nealed sample. Among the three peaks at 617, 650, and 682 $\mathrm{cm}^{-1}$ found in the second-order spectra, which correspond to $2 \mathrm{TO}, \mathrm{TO}+\mathrm{LO}$, and $2 \mathrm{LO}$, respectively, ${ }^{24}$ we have used the $2 \mathrm{LO}$ peak as a reference because it yields the most accurate intensity and width estimation for all the samples annealed at different temperatures.

Second-order Raman scattering overcomes the problems present in the analysis of the first-order Raman spectra mentioned above. On the one hand, the long-wavelength LOplasmon coupled modes do not interfere with the intensity analysis of the second-order optical peaks because the $L^{-}$ are well away from this frequency range, and the $L^{+}$modes, which may have energies in this range, are of plasmon-like character and give rise to a very broad and shallow Raman signal easily distinguishable from the second-order Raman peaks. On the other hand, the difficulty associated with the different scattering volume for the first-order LO mode between implanted and unimplanted samples discussed above is also lifted. In contrast with the first-order spectra, the second-order optical spectra of the implanted samples is very close to that of virgin $\mathrm{InP},{ }^{24}$ and for the highest annealing temperatures the intensities of the different peaks reach values around $70 \%$ of those of unimplanted samples, while the intensity of the first-order LO peak is about six times lower. This fact is an experimental proof that the scattering volume for the second-order modes comprises the volume probed by the exciting light and therefore second-order intensities can be compared.

In Fig. 3(a) we plot the intensity of the 2LO peak, normalized to the intensity of the $2 \mathrm{LO}$ peak in virgin InP, versus annealing temperature, and in Fig. 3(b) the normalized width at half height of the $2 \mathrm{LO}$ peak versus annealing temperature is shown. As can be seen from Fig. 3(a), whereas for an annealing temperature $T_{A}=300{ }^{\circ} \mathrm{C}$ the sample still remains fully amorphized, the intensity reached at $T_{A}=400{ }^{\circ} \mathrm{C}$ shows that at this temperature recrystallization has already occurred. However, it can be seen in Fig. 3(b) that for $T_{A}=400{ }^{\circ} \mathrm{C}$ the $2 \mathrm{LO}$ is significantly wider in relation to higher annealing temperatures. Therefore, although for $T_{A}=400{ }^{\circ} \mathrm{C}$ the total recrystallized scattering volume is very similar to that of the samples annealed at higher tempera- tures, the overall quality of the crystal is much less. High dislocation density, crystalline misorientation, polycrystalline regions, etc. may still be important in samples annealed at low temperatures. As can be seen from Fig. 3(b), the width of the 2LO peak reaches its minimum value for the sample annealed at $T_{A}=875{ }^{\circ} \mathrm{C}$, very close to that of unimplanted InP. The absence of a sizable broadening in the $2 \mathrm{LO}$ peak in this sample indicates that the annealing has reduced the degree of disorder to a level comparable to that of the unimplanted sample. This fact, together with the very low intensity of the forbidden TO peak already discussed, allows us to conclude that RTA at $875^{\circ} \mathrm{C}$ for $10 \mathrm{~s}$ results in implanted crystals in which the phonon coherence length is close to that of virgin InP and the original crystalline orientation is largely preserved.

So, whereas considering only the intensity of secondorder Raman peaks it might appear that the overall crystallinity is already recovered for relatively low annealing temperatures, the decrease of their widths shows that the quality of the single crystal improves with higher annealing temperatures. Thus, second-order Raman scattering provides reliable criteria to check the sample crystallinity by comparing the intensities and widths of the Raman peaks of implanted and unimplanted samples, contrary to the first-order scattering where, as already discussed, these criteria are not applicable.

Whereas about $70 \%$ of the intensity of the $2 \mathrm{LO}$ peak can be recovered by annealing at $875^{\circ} \mathrm{C}$, the $3 \mathrm{LO}$ peak was recovered to only about $40 \%$ for the same sample, and it was slightly broader than the corresponding peak of virgin InP. However, due to the low intensity of the third-order Raman scattering peaks, the determination of their intensities and, particularly, of their widths is not accurate enough to serve as a measure of sample crystallinity. Therefore, once the low intensity of the TO mode has been checked to confirm a good orientation of the recrystallized sample, the intensities and widths of the second-order Raman peaks are the best indicators of the crystallinity improvement.

The intensity of the second- and third-order Raman peaks of the samples annealed at $875^{\circ} \mathrm{C}$ indicates that the crystallinity of implanted InP samples could not be completely recovered by RTA. The proper reconstruction of the damaged InP lattice depends on the availability of both constituent atoms in the correct proportions near the interface between damaged and recrystallized regions. ${ }^{25}$ The significant phosphorus loss occurring during annealing, ${ }^{26}$ as well as the greater range of recoiling for $\mathrm{P}$ atoms compared to $\mathrm{In}$ atoms ${ }^{26}$ may be important factors preventing a greater degree of recrystallization in InP samples.

\section{CONCLUSION}

We have shown that first-order Raman scattering is not reliable to assess the lattice recovery of implanted samples after annealing, and that it is necessary to perform secondorder Raman scattering to overcome the problems posed by the assessment based solely on first-order Raman scattering. The study of the intensities and widths of the second-order Raman peaks provides the best indication of the lattice recovery. Using these criteria, we have shown that for 
$\mathrm{Si}^{+}$-implanted InP a high quality lattice recovery is achieved by performing RTA at $875^{\circ} \mathrm{C}$ for $10 \mathrm{~s}$, which corresponds with the high electronic mobility measured in these samples, in spite of the fact that the crystal was fully amorphized before annealing.

\section{ACKNOWLEDGMENT}

The authors gratefully acknowledge the Spanish Ministerio de Educación y Ciencia for financial support.

${ }^{1}$ N. Moriya, I. Brener, R. Kalish, W. Pfeiffer, M. Deicher, R. Keller, R Magerle, E. Recknagel, H. Skudlik, Th. Wichert, and H. Wolf, J. Appl. Phys. 73, 4248 (1993).

${ }^{2}$ S. Ushioda, Solid State Commun. 15, 149 (1974).

${ }^{3}$ C. S. R. Rao, S. Sundaram, R. L. Schmidt, and J. Comas, J. Appl. Phys. 54, 1808 (1983)

${ }^{4}$ K. K. Tiong, P. M. Amirtharaj, F. H. Pollak, and D. E. Aspnes, Appl. Phys. Lett. 44, 122 (1984).

${ }^{5}$ G. Burns, F. H. Dacol, C. R. Wie, E. Burnstein, and M. Cardona, Solid State Commun. 62, 449 (1987).

${ }^{6}$ M. Holtz, R. Zallen, and O. Brafman, Phys. Rev. B 38, 6097 (1988).

${ }^{7}$ J. Wagner, Appl. Phys. Lett. 52, 1158 (1988).

${ }^{8}$ J. Wagner and C. R. Fritsche, J. Appl. Phys. 64, 808 (1988).

${ }^{9}$ U. V. Desnica, J. Wagner, T. E. Haynes, and O. W. Holland, J. Appl. Phys. 71, 2591 (1992)
${ }^{10}$ R. Ashokan, K. P. Jain, H. S. Mavi, and M. Balkanski, J. Appl. Phys. 60, 1985 (1986).

${ }^{11}$ M. Holtz, R. Zallen, A. E. Geissberger, and R. A. Sadler, J. Appl. Phys. 59, 1946 (1986)

${ }^{12}$ J. Wagner and Ch. Hoffmann, Appl. Phys. Lett. 50, 682 (1987).

${ }^{13}$ H. Yoshida and T. Katoda, J. Appl. Phys. 67, 7281 (1990).

${ }^{14}$ M. Gargouri, B. Prevot, and C. Schwab, J. Appl. Phys. 62, 3902 (1987).

${ }^{15}$ L. L. Abels, S. Sundaram, R. L. Schmidt, and J. Comas, Appl. Surf. Sci. 9, 2 (1981).

${ }^{16}$ S. J. Yu, H. Asahi, S. Emura, H. Sumida, S. Gonda, and H. Tanoue, J. Appl. Phys. 66, 856 (1989).

${ }^{17}$ E. Bedel, G. Landa, R. Carles, J. B. Renucci, J. M. Roquais, and P. N. Favennec, J. Appl. Phys. 60, 1980 (1986).

${ }^{18}$ R. K. Nadella, M. V. Rao, D. S. Simons, P. H. Chi, M. Fatemi, and H. B. Dietrich, J. Appl. Phys. 70, 1750 (1991).

${ }^{19}$ R. Cuscó, G. Talamàs, L. Artús, G. González-Díaz, and J. Martin, J. Appl. Phys. 79, 3927 (1996).

${ }^{20}$ H. Shen, G. Yang, Z. Zhou, W. Huang, and S. Zou, J. Appl. Phys. 68, 4894 (1990).

${ }^{21}$ J. M. Martin, S. García, I. Martil, G. González-Díaz, R. Cuscó, and L. Artús, Mater. Sci. Technol. 11, 1203 (1995).

${ }^{22}$ D. E. Aspnes and A. A. Studna, Phys. Rev. B 27, 985 (1983).

${ }^{23}$ D. Olego and M. Cardona, Phys. Rev. B 24, 7217 (1981).

${ }^{24}$ L. Artús, R. Cuscó, J. M. Martín, and G. González-Díaz, Phys. Rev. B 50, 11552 (1994).

${ }^{25}$ L. A. Christel and J. F. Gibbons, J. Appl. Phys. 52, 5050 (1981).

${ }^{26}$ A. Dodabalapur and B. G. Streetman, J. Electron. Mater. 18, 65 (1989). 